\title{
MATRIX REPRESENTATION OF SIMPLE HALFRINGS BY
}

H. E. STONE

\begin{abstract}
The structure of halfrings which are strong direct sums of minimal subtractive right ideals is studied. A class of right simple hemirings which contains both division hemirings and differential subsemirings of division rings is introduced and studied extensively as a tool in this investigation. A matrix representation is obtained for a class of halfrings which properly includes differential subsemirings of simple Artinian rings.
\end{abstract}

1. Introduction. A semiring is a triple $(H,+, \cdot)$ such that $(H,+)$ is a semigroup with identity $0,(H, \cdot)$ is semigroup with zero 0 , and multiplication distributes over addition. If the addition is commutative, $H$ is called a hemiring. A subsemiring $S$ of a hemiring $H$ is differential in $H$ if $\forall h \in H \exists s$, $t \in S$ with $s+h=t$. A halfring is a hemiring $H$ which is differential in some ring $\bar{H}$, which is then uniquely determined. This paper continues the point of view of [9], and terminology introduced there will be extended without comment to hemirings generally.

A hemiring is [right, left] simple if it has no nontrivial subtractive [right, left] ideals. It is [right, left] ideal-free if it has no nontrivial [right, left] ideals. A division semiring is a semiring with $1 \neq 0$ all of whose nonzero elements have multiplicative inverse. It is clear that division hemirings may be characterized among nonzero hemirings as those which are right and left ideal-free, and among hemirings with identity as those which are right ideal-free. Grillet and Grillet [4], [5] have recently studied ideal-free and semisimple semirings very successfully using the techniques of semigroup theory. Earlier, Bourne [1], Bourne and Zassenhaus [2], [3], Steinfeld [8], and Wiegant [10] had studied semirings which are direct sums of minimal right ideals. The culmination of that study is an analogue of the Wedderburn theorem which may be paraphrased as follows:

An ideal-free semiring with identity which is a strong direct sum of finitely many minimal right ideals is isomorphic to a full semiring of matrices over a division semiring.

Presented to the Society, January 23, 1975; received by the editors September 24, 1973 and, in revised form, March 15, 1977.

AMS (MOS) subject classifications (1970). Primary 16A78; Secondary 16A40, 16A42.

Key words and phrases. Hemiring, halfring, entire, cancellative, simple, semisimple, Artinian, division semiring, full matrix halfring.

- American Mathematical Society 1977 
Here a semiring is said to be a direct sum of a family $\left\{S_{\alpha}\right\}$ of subsemirings if it is the sum of the subsemirings and 0 has a unique representation. In case $\mathcal{P}$ is a property of halfrings which is inherited by differential subsemirings, we adopt as a general principle the terminology that $H$ is strongly $\mathcal{P}$ if and only if $\bar{H}$ is $\mathscr{P}$. In particular, a halfring $H$ is a strong direct sum of subsemirings $\left\{S_{\alpha}\right\}$ if and only if $\bar{H}$ is a direct sum of subrings $\left\{\bar{S}_{\alpha}\right\}$. It is easy to see that $H$ is a strong direct sum of the $\left\{S_{\alpha}\right\}$ if and only if every element of $H$ has a unique representation. We then take this condition as the definition of strong direct sum for general semirings.

It is the object of this paper to analyze the structure of hemirings which are strong direct sums of minimal subtractive right ideals, with particular attention to the possibility of obtaining matrix representations. It is easy to see that because of the unique representation of elements, a strong summand of a hemiring must be subtractive. It is considerably less restrictive, however, to require minimality only among subtractive right ideals, as well as being more natural as a generalization of the ring theory case.

2. Nilpotent ideals and semi-isomorphisms. In what follows, we will often have to adapt to hemirings arguments based on the absence of nilpotent ideals. We will also obtain some results involving semi-isomorphic representations. Hence we collect here some information regarding the behavior of these concepts in general hemirings.

Semi-isomorphisms are not very promising tools for structural investigation, and the author devoted considerable attention in [9] to determining when they could be strengthened to isomorphisms. The following result is a dramatic illustration of how little information a semi-isomorphism can reveal. Construct the free hemiring on a set $S$ as the free Abelian monoid, written additively, over the free multiplicative semigroup on $S$, extending the multiplication by distributivity. This is clearly a halfring, and it is easy to see that every hemiring is a homomorphic image of a free halfring. But it is also easy to see that if $K$ is any subtractive ideal of a halfring $H$, then $H / K$ is a halfring. Hence

\section{Proposition 1. Every hemiring is a semi-isomorphic image of a halfring.}

Hence a semi-isomorphic image of a nice structure need not retain any nice properties. However, the requirement that a hemiring have a semi-isomorphic image with nice properties is generally a much greater restriction. The best illustration of this is LaTorre's observation [7] that a semiring semiisomorphic to a ring is itself a ring. Another example is the situation dual to Proposition 1: the requirement that a hemiring be semi-isomorphic to a halfring. If $\mathscr{P}$ is a property of hemirings, we adopt as a general principle the terminology that $H$ is pre- $\odot$ if and only if $H$ has a semi-isomorphic image 
with $\mathscr{P}$. Hence we are discussing the class of prehalfrings. Bourne and Zassenhaus [3] introduced the zeroid $Z(H)=\{z \in H: x+z=x$ for some $x \in H$ \} of a hemiring $H$, and lizuka [6] showed that $H / Z$ is semi-isomorphic to a halfring. Hence the prehalfrings are exactly the hemirings with zeroid 0 . This is a substantial restriction compared to the liberty allowed by Proposition 1.

Following Bourne and Zassenhaus we define a hemiring to be potent if no nonzero one-sided ideal is nilpotent. However, we wish to impose conditions only on subtractive ideals. If $A$ and $B$ are right ideals in a hemiring, $A B$ is again a right ideal, but need not be subtractive even if $A$ and $B$ are. We denote by $\langle S\rangle$ the subtractive subset of $H$ generated by any additively closed subset $S$. The following result, whose proof consists of routine computations, reconciles these two viewpoints.

Proposition 2. Let $A$ and $B$ be subsemirings of a hemiring $H$. Then $\langle A B\rangle=\langle\langle A\rangle B\rangle=\langle A\langle B\rangle\rangle=\langle\langle A\rangle\langle B\rangle\rangle$.

Corollary A. If $A$ is a right ideal of $H$, then $A^{n}=0$ if and only if $\langle A\rangle^{n}=0$.

It is known that the sum of all nilpotent right ideals of a hemiring $H$ is a two-sided nil ideal $p(H)$ containing all nilpotent left ideals of $H$.

Corollary B. $p(H)$ is a subtractive ideal of $H$.

Corollary C. Every potent halfring is semi-isomorphic to a strongly potent halfring.

Proof. If $I$ is a nilpotent ideal of $\bar{H}$, then $I \cap H$ is a nilpotent subtractive ideal of $H$. Hence $p(\bar{H}) \cap H=p(H)$. If $p(H)=0$, then $p(\bar{H})$ belongs to the type $\tau(0)$ of 0 as studied in [9], and hence there is some maximal member $M \in \tau(0)$ with $p(\bar{H}) \subseteq M$. The natural homomorphism $\nu$ of $H$ into $\bar{H} / M$ is a semi-isomorphism. Then $p(H \nu)=0$, and $p(\bar{H} \nu) \in \tau(0)$ as above. But by the maximality of $M, \tau(0)$ in $\bar{H} / M$ consists of 0 only, so that $p(\bar{H} \nu)=0$. Hence $H \nu$ is a strongly potent halfring.

3. Halffields. The most natural place to look for semirings having matrix representations is among subsemirings of potent right Artinian rings. An extension of what we have just done gives

Proposition 3. Every simple potent strongly right Artinian halfring is semiisomorphic to a differential subsemiring of the full ring of matrices over a field.

Proof. Since $p(\bar{H}) \in \tau(0)$, there is a maximal ideal $I$ of $\bar{H}$ in $\tau(0)$, containing $p(\bar{H})$, and $\bar{H} / I$ is a simple, potent Artinian ring, and hence a full ring of matrices over a field (division ring). The natural homomorphism is a 
semi-isomorphism carrying $H$ onto a differential subsemiring of $\bar{H} / I$. Q.E.D.

If $H$ is a hemiring, we denote the hemiring of all $n \times n$ matrices with entries in $H$ by $M_{n}(H)$. If $a \in M_{n}(H)$, we denote the $(i, j)$ th entry of $a$ by $a(i, j)$. It is easy to see

PROPOSITION 4. If $S$ is a differential subsemiring of a hemiring $H$, then $M_{n}(S)$ is differential in $M_{n}(H)$. In particular, if $H$ is a halfring, so is $M_{n}(H)$, and $M_{n}(H)^{-}=M_{n}(\bar{H})$.

Unfortunately we cannot expect to get a full matrix halfring in general, for many other kinds of differential subsemirings occur in matrix rings.

EXAMPLe 5. Let $H=\{0\} \cup\left\{a \in M_{2}(F): a(i, j)<i j\right\}$. Then $H$ is differential in the full matrix halfring over the nonnegative rationals $F$, but $H$ is not a full matrix semiring over any semiring.

Note that Example 5 has no direct summand. We can hope for a more pleasant situation when we ask that $H$ be a strong direct sum of minimal subtractive ideals $H_{1}, \ldots, H_{n}$. Then $\bar{H}$ is the direct sum of $\bar{H}_{1}, \ldots, \bar{H}_{n}$. However, $\bar{H}_{i}$ may not be a minimal right ideal of $\bar{H}$.

We will say that $M$ is a strongly minimal subtractive right ideal of a halfring $H$ if $\bar{M}$ is a minimal right ideal of $\bar{H}$. If $\bar{H}$ is a potent right Artinian ring, $\bar{M}$ is generated by a right identity $e$, and $e \bar{M} e$ is a field. It is easy to see that $e M e$ is a differential subsemiring of the field $e \bar{M} e$. In any hemiring $H$, we say that an ordered pair $(u, v)$ is right unital if $h u+h=h v$ for all $h \in H$. Hence in a unital halfring, $v-u=1$. Finally, we define a halffield to be a halfring $H$ such that $\bar{H}$ is a field.

PROPOSITION 6. Let $H$ be a strongly potent halfring and $M$ a subtractive right ideal of $H$ with right unital pair $(r, s)$. Then $M$ is strongly minimal if and.only if $(s-r) M(s-r)$ is a halffield.

The nonnegative rationals $F$ are both a halffield and a division halfring. The example constructed in [9] is a division halfring which is not a halffield. We now present some examples of halffields which are not division semirings.

EXAMPLE 7. Let $r>1$ be a real number, and let $F_{r}=\{x \in F: r<x\} \cup$ $\{0\}$. Then $F_{r}$ is a subhalffield of the rational numbers $\bar{F}$, but no $F_{r}$ is a division halfring since none even has an identity.

Proposition 8. (a) If $H$ is a halffield, $M_{n}(H)$ is strongly simple, strongly potent, and is a strong direct sum of strongly minimal subtractive right ideals.

(b) If a strongly simple, strongly potent halfring $H$ is a strong direct sum of strongly minimal subtractive left ideals, then $H$ is differential in a full ring of matrices over a field. 
However, the gap between full matrix halfrings and differential subsemirings of full matrix rings cannot be closed even in this case.

EXAMPLE 9. Let $H=\left\{a \in M_{2}(F): a(i, j) \in F_{j}\right\}$. Then $H$ is strongly simple, strongly potent and is a strong direct sum of the strongly minimal right ideals $H_{1}=\{a \in H: a(i, 1)=0\}$ and $H_{2}=\{a \in H: a(i, 2)=0\}$. Nevertheless $H$ is not a full matrix halfring over any semiring.

This is not what we could wish, but since the subsemiring is differential, it is closely related to the full matrix ring. This is an additive analogue of the representation of a ring as an order in a matrix ring, and it is probably as much as can be expected in this generality. In what follows, we will be seeking ways to weaken the requirement that the summands be strongly minimal, and correspondingly the requirement that the halfring be strongly right Artinian. Nevertheless, the halffields are an interesting and important class of semirings, and we make a beginning on their study by classifying the subhalffields of the rationals. This case is made accessible by the fact that a proper subsemiring of the rationals must be contained in the nonnegative rationals $F$, and the fact that since the rationals are a prime field, a subhalffield must be differential.

THEOREM 10. A subsemiring $S$ of the nonnegative rationals $F$ is a halffield if and only if for each prime $p$ there exists $n(p) \in N$, the nonnegative integers, such that $p \nmid n(p)$ and $p^{-1} n(p) \in S$.

Proof. If $p$ divides no denominator of a fraction in $S$, it divides no denominator of a fraction in $\bar{S}$; hence $\bar{S} \neq \bar{F}$. Thus $m n^{-1} \in S$ for some $m$, $n \in N$, where $(m, n)=1$ and $p \mid n$. Then $n p^{-1} \in N$, so that $m p^{-1} \in S$. Then $p \nmid m$, and we take $n(p)=m$.

Conversely, let $S$ be a subsemiring of $F$ satisfying the condition. It suffices to show $\bar{S}=\bar{F}$, and for this it suffices to show that if $0 \neq n \in N$, then $n^{-1} \in \bar{S}$. Let $I=\{n \in N: 0 \neq n \in \bar{S}\}$. Since $p^{-1} n(p) \in S$ for any prime $p$, $n(p) \in S \subseteq \bar{S}$, so that $I \neq \varnothing$. Let $m$ be the least member of $I$. If $m>1$, there is a prime $q$ such that $q \mid m$, and $q t=m$ for some $t \in N, 0<t<m$. Since $q \nmid n(q)$, there are $r, s \in \bar{N}$ with $r n(q)+s q=1$. Hence $r n(q) t+s q t=$ $t=r \operatorname{rn}(q)+s m \in \bar{S}$, which contradicts the minimality of $m$. Hence $m=1$ $\in \bar{S}$.

Suppose for induction that $n^{-1} \in \bar{S}$ for $0<n<k$, where $k>1$. If $k$ is not prime, $k=g h$, where $1<g, h<k$. Then $h^{-1}, g^{-1} \in \bar{S}$, so that $g^{-1} h^{-1}$ $=k^{-1} \in \bar{S}$. If $k$ is prime, let $m$ be the greatest integer $<k^{-1} n(k) \in S$. Then $k^{-1} n(k)-m=k^{-1} n^{\prime}(k) \in \bar{S}$, where $0<n^{\prime}(k) \leq k$. But then $n^{\prime}(k)^{-1} \in \bar{S}$, so that $k^{-1} \in \bar{S}$ also. Hence by induction, $\bar{S}=\bar{F}$ and $S$ is a subhalffield of F. Q.E.D. 
4. Entire hemirings. We seek a substitute for the halffields which will be associated with minimal subtractive right ideals in potent halfrings. The success of division halfrings and halffields suggests that what we want is a common generalization of these classes, and the basic nature of fields in ring theory suggests that conditions of right and left simplicity ought to be involved.

We define the right annihilator of a set $S \subseteq H$ as $\rho(S)=\{h \in H: S h=$ $0\}$. Clearly $\rho(S)$ is a subtractive left ideal, and if $S$ is a right ideal, $\rho(S)$ is a two-sided ideal. For $a, b$ in a halfring $H$, we denote by $\rho(a, b)$ the set $H \cap \rho(a-b)$, where $\rho(a-b)$ is the right annihilator of $a-b$ in $\bar{H}$. We make similar definitions for the left annihilators $\lambda(S), \lambda(a, b)$. Finally, we define a hemiring to be entire if it is free of zero-divisors.

PropositIon 11. Every nonzero right simple hemiring is entire.

Proof. Let $x y=0, x \neq 0$. Then $x \in \lambda(y)$, so that $\lambda(y)=H$. Hence $y \in \rho(H)$, and since $H^{2} \neq 0, \rho(H) \neq H$. Then $\rho(H)=0$, so that $y=0$. Q.E.D.

In order to determine the nature of right simple hemirings, we need to learn the effect of being entire. The class of entire hemirings has the attractive property that for any semi-isomorphism $\sigma, H$ is entire if and only if $H \sigma$ is entire. However, the absence of subtraction means that entire hemirings do not enjoy the benefits of multiplicative cancellation as is the case for rings. Example 5 is an entire halfring which is not right or left cancellative. Even worse behavior can occur, as in the following example.

EXAMPLE 12. Let $H$ be the set of polynomials over the nonnegative integers $N$ with degree at most 1 . With the polynomial addition and the multiplication given by $(a x+b)(c x+d)=(a+b)(c+d), H$ is a commutative entire halfring having no multiplicatively cancellative element.

PROOF. It is easy to check that $H$ is a commutative halfring. Since $a, b, c, d>0,(a x+b)(c x+d)=(a+b)(c+d)=0$ implies $a x+b=0$ or $c x+d=0$, and $H$ is entire. For fixed $p x+q \in H,(a x+b)(p x+q)=$ $(c x+d)(p x+q)$ implies $(a+b)(p+q)=(c+d)(p+q)$, and if $p+q \neq$ $0, a+b=c+d$. For any choice of $a, b$ not both 0 there exists at least one choice of $c, d$ with $(a, b) \neq(c, d)$ and $a+b=c+d$. Hence no element of $H$ is cancellative. Q.E.D.

The next example shows that the equivalence of right and left cancellation also fails in halfrings.

EXAMPLE 13. Let $H$ consist of the polynomials in noncommuting indeterminates $x$ and $y$ over the nonnegative integers, with the usual addition, but with multiplication modified by the condition that $y x=y$. Then $H$ is a right cancellative halfring which is not left cancellative. 
Proof. Since $y x=y \cdot 1, y$ is not left cancellable. To prove right cancellation, observe that multiplication of monomials is right cancellative and extend by a straightforward induction. Q.E.D.

Finally, the example constructed in [9] is a division halfring, hence cancellative, whose ring of differences is not cancellative.

Proposition 14. Let $H$ be a halfring. Then $\bar{H}$ is cancellative if and only if $H$ satisfies the condition that $a c+b d=a d+b c$ imply $a=b$ or $c=d$.

It is then consistent with our terminology to call a hemiring strongly cancellative if it satisfies this condition.

The misbehavior of multiplication in entire hemirings must be global in some sense. We borrow from semigroup theory the definition that a hemiring $H$ is right reductive if when $a h=b h$ for each $h \in H$, then $a=b$. If $H$ has even one right cancellative element, or if $H$ is a right unital halfring, $H$ is right reductive.

Proposition 15. Let $H$ be a nonzero left simple halfring. If $H$ is right reductive it is right cancellative.

Proof. Suppose $a x=b x$ for $0 \neq x$. Then $x \in \rho(a, b) \neq 0$, and $\rho(a, b)=$ $H$ by the left simplicity of $H$. But then $a=b$ by the right reductivity. Q.E.D.

We recall that if $H$ is potent, $x H=0$ implies $x=0$. Hence we have reductivity automatically in potent rings.

Proposition 16. Let $H$ be a strongly potent halfring. Then $H$ is reductive.

Proof. By hypothesis, $\bar{H}$ is potent. If $a, b \in H$ with $a h=b h$ for every $h \in H$, then $(a-b) x=0$ for every $x \in \bar{H}$. Hence $a-b=0$ and $a=b$. Left reductivity follows by a similar argument. Q.E.D.

COROLlaRY A. Every potent prehalfring is semi-isomorphic to a reductive halfring.

Proof. There is a semi-isomorphism $\sigma_{1}$ such that $H \sigma_{1}$ is a halfring. But it is evident that semi-isomorphisms preserve potency. Then there is a further semi-isomorphism $\sigma_{2}$ onto a strongly potent halfring, and $\sigma_{1} \sigma_{2}$ is the desired semi-isomorphism. Q.E.D.

COROLLARY B. Every nonzero left simple prehalfring is semi-isomorphic to a right cancellative halfring.

These results suggest that we might obtain cancellative representations of entire semirings in the hemiring case as well. We say that a hemiring $H$ has the common right multiple property if for every nonzero $a, b \in H$ there exist nonzero $x, y \in H$ with $a x=b y$. 
Proposition 17. Let $H$ be an entire hemiring with the common right multiple property. Then $H$ is semi-isomorphic to a right cancellative hemiring.

Proof. Define a congruence on $H$ by $a \equiv b$ if and only if $a x=b x$ for some nonzero $x \in H$. Since $H \neq 0, a \equiv a$. If $a \equiv b$ and $b \equiv c$, with $a x=$ $b x, b y=c y$, choose nonzero $x^{\prime}, y^{\prime} \in H$ with $x x^{\prime}=y y^{\prime}$. Then $a x x^{\prime}=b x x^{\prime}=$ $b y y^{\prime}=c y y^{\prime}$, and $a \equiv c$. Clearly $\equiv$ is symmetric and compatible with addition and left multiplication. Let $a \equiv b$ with $a x=b x$ and suppose $c \neq 0$. Choose nonzero $c^{\prime}, x^{\prime} \in H$ with $c c^{\prime}=x x^{\prime}$. Then $a c c^{\prime}=a x x^{\prime}=b x x^{\prime}=b c c^{\prime}$, so that $a c \equiv b c$. Hence the congruence classes form a hemiring $H^{\prime}$, and the natural mapping $\sigma: H \rightarrow H^{\prime}$ is a semi-isomorphism. If $a \sigma x \sigma=b \sigma x \sigma$ with $x \sigma \neq 0$, then $a x \sigma=b x \sigma$ and $a x x^{\prime}=b x x^{\prime}$ for some $x^{\prime} \neq 0$. Then $\dot{x} x^{\prime} \neq 0$, so that $a \sigma=b \sigma$. Hence $H \sigma$ is right cancellative. Q.E.D.

COROLlARY A. If $H$ is an entire hemiring with both the right and left common multiple properties, $H$ is semi-isomorphic to a cancellative hemiring. Further, if $H$ is a halfring, the image may be chosen to be a halfring also.

Proof. By the dual result, there is a semi-isomorphism $\sigma_{1}$ of $H$ onto a left cancellative hemiring. If $a \sigma_{1}, b \sigma_{1}$ are nonzero, so are $a, b$, and there exist $a^{\prime}, b^{\prime} \in H$ with $a a^{\prime}=b b^{\prime}$. Then $a \sigma_{1} a^{\prime} \sigma_{1}=b \sigma_{1} b^{\prime} \sigma_{1}$, and $a^{\prime} \sigma_{1}, b^{\prime} \sigma_{1}$ are nonzero. Hence $H \sigma_{1}$ has the right common multiple property, and there is a semi-isomorphism $\sigma_{2}$ such that $H \sigma_{1} \sigma_{2}$ is right cancellative. Let $a x=a y$ in $H \sigma_{1} \sigma_{2}$, with $a \neq 0$. Let $a^{\prime} \neq 0, x^{\prime}, y^{\prime} \in H \sigma_{1}$ so that $a^{\prime} \sigma_{2}=a, x^{\prime} \sigma_{2}=x$, $y^{\prime} \sigma_{2}=y$. Then $a^{\prime} x^{\prime} z=a^{\prime} y^{\prime} z$ for some $z \in H \sigma_{1}$, so that $x^{\prime} z=y^{\prime} z$ and $x=y$ by left cancellation in $H \sigma_{1}$. Hence $H \sigma_{1} \sigma_{2}$ is cancellative. It is clear that these congruences preserve additive cancellation when it is present. Q.E.D.

5. Reciprocal hemirings. We wish to consider a substitute for the existence of inverses which will make sense in a general hemiring. In order to avoid technicalities analogous to left inverses with respect to right identities, we will consider in this section only hemirings having a two-sided unital pair. A left inversion of $x \in H$ is an ordered pair $(a, b)$ such that $(a x, b x)$ is unital; $x$ is called a right reciprocal if it has a left inversion. If every nonzero element of $H$ is a right reciprocal, we call $H$ a right reciprocal hemiring. We extend these definitions to dual and two-sided concepts, but caution that an element which is both right and left reciprocal need not be two-sided reciprocal. However this is the case if $H$ is a halfring. Plainly $H$ is a right reciprocal halfring if and only if every nonzero element of $H$ is a right unit in $\bar{H}$. Hence every right reciprocal of a halfring is left cancellable.

THEOREM 18. Let $H$ be a unital hemiring. Then $H$ is right simple if and only if it is right reciprocal. 
Proof. Suppose $H$ is right reciprocal, and let $K$ be a subtractive right ideal of $H$. For $0 \neq k \in K$, let $(a, b)$ be a left inversion, so that $(a k, b k)$ is unital. But $a k, b k \in K$; hence $K=H$.

Conversely, let $H$ be right simple, and let $0 \neq x \in H$. Since $H$ is unital it is nonzero, and by Proposition 11 it is entire. Then $H x$ is a nonzero right ideal, and $H x$ must be differential in $H$. Thus there are $a, b$ in $H$ with $a x+u=b x$ and $a^{\prime}, b^{\prime}$ in $H$ with $a^{\prime} x+v=b^{\prime} x$. Thus $\left(a^{\prime}+b\right) x=a^{\prime} x+b x=a^{\prime} x+a x$ $+u$ and $\left(a+b^{\prime}\right) x=a x+b^{\prime} x=a x+a^{\prime} x+v$. It is easy to verify that for every $h \in H,(u+h, v+h)$ is unital. Hence $\left(a^{\prime}+b, a+b^{\prime}\right)$ is a left inversion of $x$. Q.E.D.

Proposition 19. Every unital right cancellative right Artinian hemiring is right reciprocal.

Proof. Let $K$ be a minimal subtractive right ideal of $H$, and let $0 \neq x \in$ $K$. Then $H x^{2} \subseteq K$ is a nonzero right ideal of $H$, hence differential in $K$. Let $a, b \in H$ such that $a x^{2}+x=b x^{2}$. Then for each $h \in H, h a x^{2}+h x=h b x^{2}$, and cancelling $x$ gives $h a x+h=h b x$. Since $h a x, h b x \in K, H=K$. Q.E.D.

COROLlary A. If $H$ is a unital entire right Artinian hemiring with the right common multiple property, $H$ is semi-isomorphic to a right reciprocal hemiring.

Proposition 20. Let $H$ be a right reciprocal hemiring. Then the hemiring $M_{n}(H)$ of $n \times n$ matrices over $H$ is simple.

Proof. For $0 \neq h \in H$ and $1 \leqslant p, q \leqslant n$, define $e_{p q} \in M_{n}(H)$ by $e_{p q}(i, j)$ $=h$ for $(i, j)=(p, q), 0$ otherwise. Let $K$ be a subtractive ideal of $M_{n}(H)$, with $0 \neq e \in K$. Then $e(p, q) \neq 0$ for some $p, q$. For $1 \leqslant k \leqslant n$ define $f_{k}=e_{k p} e e_{q k}$. Then $f_{k}(i, j)=0$ for $(i, j) \neq(k, k) ; f_{k}(k, k)=h e(p, q) h \neq 0$. Let $\left(a_{k}, b_{k}\right)$ be a left inversion of $f_{k}(k, k)$, and define $u_{k}, v_{k} \in M_{n}(H)$ by $u_{k}(i, j)=0=v_{k}(i, j)$ for $(i, j) \neq(k, k) ; u_{k}(k, k)=a_{k}, v_{k}(k, k)=b_{k}$. Then $u=\Sigma u_{k} f_{k} \in K, v=\Sigma v_{k} f_{k} \in K$ since $K$ is a two-sided ideal. But clearly $(u, v)$ is a unital pair for $M_{n}(H)$, and since $K$ is subtractive, $K=M_{n}(H)$. Q.E.D.

We now ask about the relationship of right reciprocal halfrings and halffields. In the commutative case this is easy to answer.

Proposition 21. A commutative nonzero halfring is a halffield if and only if it is strongly simple.

COROLlaRY A. A commutative reciprocal prehalfring is semi-isomorphic to a halffield.

Proof. Clearly a semi-isomorphism preserves the property of being a right or left reciprocal. Hence $H$ is semi-isomorphic to a commutative reciprocal 
halfring. There is a further semi-isomorphism onto a strongly simple halfring, which is in this case a halffield. Q.E.D.

It is natural to ask whether only reciprocal halfrings can be so represented. The following example shows that this is not the case.

EXAMPLE 22. Let $H=\{(0,0)\} \cup\{(a, b) \in F \times N: a>0, b>0\}$. Then $H$ is a halfring with $\bar{H}=\bar{F} \times \bar{N}$, hence not reciprocal. But the projection of $H$ onto $F$ is a semi-isomorphism.

In the noncommutative case it is not sufficient to know that 0 is monotypic. But in [9] it is observed that the condition of being semisubtractive is much stronger even than having all ideals monotypic.

Proposition 23. A semisubtractive right reciprocal halfring is a division halfring.

Proof. Let $0 \neq x \in H$, and let $(a, b)$ be a left inversion of $x$ in $H$. Then $(a x, b x)$ is unital, and $b x-a x=1 \in \bar{H}$. By semisubtractivity, either $a=b$ $+c$ or $a+c=b$ for some $c \in H$. If $a=b+c$, then $c x=(a-b) x=-1$ $\in H$, and $H$ is a ring, hence a division ring. If $a+c=b$, then $c x=(b-$ $a) x=1 \in H$, so that $x$ has a left inverse in $H$. If $H$ is not a ring, this case must hold for every $0 \neq x \in H$. Then the inverses are two-sided and $H$ is a division halfring. Q.E.D.

A similar thing occurs if $H$ is even subtractively embedded in a division hemiring.

Proposition 24. Let $S$ be a subtractive subsemiring of a division hemiring $\boldsymbol{H}$. If $S$ is right reciprocal, then $S$ is a division hemiring.

Proof. Let $0 \neq x \in S$, and let $(a, b)$ be a left inversion of $x$ in $S$. Then $(a x, b x)$ is unital in $S$, so that $a x^{2}+x=b x^{2}$. But $x$ has a (two-sided) inverse $x^{-1}$ in $H$, and

$$
a x^{2}\left(x^{-1}\right)^{2}+x\left(x^{-1}\right)^{2}=b x^{2}\left(x^{-1}\right)^{2} .
$$

Hence $a+x^{-1}=b$, and since $S$ is subtractive in $H, x^{-1} \in S$. Q.E.D.

It is reasonable to ask when a right reciprocal hemiring is also left reciprocal.

Proposition 25. Let $H$ be a right reciprocal hemiring. If $H$ is cancellative, it is reciprocal.

Proof. Let $(a, b)$ be a left inversion of $x \in H$. Then $(a x, b x)$ is unital and $x a x+x=x b x$. For every $h \in H, h x a x+h x=h x b x$, and cancelling $x$ gives $h x a+h=h x b$. Thus $(x a, x b)$ is right unital. Now cancelling on the other side shows $(x a, x b)$ is unital; hence $x$ is a reciprocal. Q.E.D.

COROLlary A. A cancellative unital right Artinian hemiring is reciprocal. 
COROLlary B. A unital right Artinian hemiring with right and left common multiple property is semi-isomorphic to a reciprocal hemiring.

Clearly for halfrings cancellativity is also necessary. However a very important but different condition will suffice. We say that $(u, v)$ is a primitive right unital pair for a halfring $H$ if it is a right unital pair and $v-u$ is the unique nonzero idempotent of $(v-u) \bar{H}(v-u)$.

PROPOSITION 26. If $H$ is a right reciprocal halfring with a primitive unital pair, then $H$ is reciprocal.

PRoof. For $0 \neq x \in H$, there is an $r \in \bar{H}$ with $r x=1$. Since $(x r)^{2}=x r x r$ $=x r$, by hypothesis $x r=1$. Q.E.D.

We will call a reciprocal halfring with a primitive unital pair a parafield. We present some examples to show the position of this concept.

EXAMPLe 27. Let $H=\{(0,0)\} \cup\{(x, y) \in F \times F: x, y>1\}$. Then $H$ is a halfring with $\bar{H}=\bar{F} \times \bar{F}$; hence $H$ is a reciprocal halfring which is not a parafield.

EXAMPLE 28. Let $H$ be the halfring of formal power series with nonnegative rational coefficients, and let $P=\left\{p \in H: p_{0}>0\right\} \cup\{0\}$. Then $P$ is a differential halfring of $H$ and every nonzero element of $P$ is a unit in $\bar{P}$. Since $\bar{P}$ is not a field, $P$ is not a halffield; but $\bar{P}$ is cancellative, so that $P$ is clearly a parafield.

It should be noted that although the class of reciprocal halfrings includes all division halfrings, this is no longer true of parafields. However, every halffield is a parafield, and we now investigate conditions when this can be reversed.

PROPOSITION 29. Let $H$ be a right reciprocal halfring which is not a ring of finite characteristic. Then $\bar{H}$ is a module over the rationals $\bar{F}$.

Proof. Since $H$ is unital, $1 \in \bar{H}$, and clearly $\bar{H}$ contains a copy of the integers $\bar{N}$. Let $0 \neq x \in H$ and let $n>0$. Let $r \in \bar{H}$ be a left inverse for $n x \in H$. (Note that if $n x=0$ for some $n>0$, then $H$ is equal to the ideal of all its additively invertible elements, hence a ring of finite characteristic.) Since $n$ is central, $r x$ is a two-sided inverse for $n$, and thus is uniquely determined. Then clearly $\bar{H}$ contains a copy of the rationals $\bar{F}$. Since $\bar{F}$ is a subring of $\bar{H}, \bar{H}$ is an $\bar{F}$-module. Q.E.D.

Let the dimension of $\bar{H}$ as a vector space over $\bar{F}$ be called the rank of $H$.

COROLLARY A. Let $H$ be a right reciprocal halfring of finite rank. If $H$ is strongly cancellative, it is a halffield.

Proof. Since $\bar{H}$ is cancellative, $H$ is a parafield. But since $\bar{H}$ has finite dimension over $\bar{F}$, it is a field. Q.E.D. 
6. Matrices over parafields. We now show that the class of right reciprocal halfrings is the correct class for our purposes.

THEOREM 30. Let $H$ be a halfring, and let $M$ be a potent subtractive right ideal of $H$ with a right unital pair $(r, s)$. Then $M$ is a minimal subtractive right ideal of $H$ if and only if $(s-r) M(s-r) \subseteq \bar{M} \subseteq \bar{H}$ is a right reciprocal halfring.

Proof. Let $s-r=e \in \bar{M}$. Then $e$ is a right identity of $\bar{M}$ and $e M e$ is a subsemiring of $\bar{M}$ with $(e M e)^{-}=e \bar{M} e$. Since $\bar{H} e \subseteq \bar{M}=\bar{M} e, e \bar{M} e=e \overline{H e}$. Clearly $e$ is a two-sided identity for $e \bar{M} e$.

If $M$ is minimal and $0 \neq c \in e M e$, then $c=e m e$ for some $m \in M$. Since $0 \neq e m \in \bar{M} m, M c=M e m e=M m \neq 0$. Now $M m$ is a right ideal of $H$, hence differential in $M$. Thus $\bar{M} m=\bar{M}$, and $e \bar{M} e=e \bar{M} e m e=(e \bar{M} e) c$. Then $c$ is a right unit in $e \bar{M} e$, and hence $e M e$ is right reciprocal.

Conversely, let $e M e$ be a right reciprocal halfring, and suppose $M^{\prime} \subseteq M$ is a nonzero subtractive right ideal of $H$. If $e M^{\prime}=0$, then $M^{\prime} M^{\prime} \subseteq M M^{\prime}=$ $M e M^{\prime}=0$. Since $M^{\prime}$ is also a right ideal of $M$, this contradicts the potency of $M$; hence $0 \neq e m^{\prime}$ for some $m^{\prime} \in M^{\prime}$. Now $e m^{\prime}=e m^{\prime} e$ has a left inverse $a \in e \bar{M} e$, whence $e=a e m^{\prime} e \in \bar{H} m^{\prime} e \subseteq \bar{M}^{\prime}$. Hence $\bar{M}^{\prime}=\bar{M}$, and $M$ is indeed minimal. Q.E.D.

Unfortunately, right reciprocal halfrings do not seem to have sufficient strength to force the matrix representation result we are seeking. It is for this reason that we have introduced parafields.

Proposition 31. Let $H$ be a halfring. For $i=1,2$ let $H_{i}$ be a minimal subtractive right ideal of $H$ with primitive right unital pair $\left(u_{i}, v_{i}\right)$, and let $e_{i}=v_{i}-$. If $H_{1} H_{2} \neq 0$, there exist elements $S_{12} \in e_{1} H e_{2}$ and $S_{21}^{\prime} \in e_{2} \bar{H} e_{1}$ with $S_{12} S_{21}^{\prime}=e_{1}$ and $S_{21}^{\prime} S_{12}=e_{2}$.

Proof. Since $H_{1}^{2}$ is differential in $H_{1}, H_{1}^{2} H_{2} \neq 0$. Let $h_{i} \in H_{i}$ with $H_{1} h_{1} h_{2}$ $\neq 0$. Since $H h_{1} h_{2} \subseteq H_{2}$ is a right ideal of $H, \bar{H}_{2}=\left(H h_{1} h_{2}\right)^{-}=\bar{H} h_{1} h_{2}$, and $e_{2} \bar{H} h_{1} h_{2}=e_{2} \bar{H}=\left(e_{2} H e_{2}\right)^{-}$. Then there exists $r \in \bar{H}$ with $0 \neq e_{2} r h_{1} h_{2} \in$ $e_{2} \mathrm{He}_{2}$, and hence there exists $r^{\prime} \in e_{2} \bar{H} e_{2}$ with $r^{\prime}\left(e_{2} r h_{1} h_{2} e_{2}\right)=e_{2}$. Let $S_{12}=$ $e_{1} h_{2} e_{2}, S_{21}^{\prime}=r^{\prime} r h_{1} \in e_{2} \bar{H} e_{1}$. Then $S_{21}^{\prime} S_{12}=r^{\prime} r h_{1} e_{1} h_{2} e_{2}=e_{2}$. Now $S_{12} S_{21}^{\prime} \in$ $e_{1} \bar{H} \dot{e}_{1}$, and $S_{12} S_{21}^{\prime} \neq 0$. Since

$$
\left(S_{12} S_{21}^{\prime}\right)^{2}=S_{12}\left(S_{21}^{\prime} S_{12}\right) S_{21}^{\prime}=S_{12} e_{2} S_{21}^{\prime}=S_{12} S_{21}^{\prime}
$$

and $e_{1}$ is the only idempotent of $e_{1} \bar{H} e_{1}, S_{12} S_{21}^{\prime}=e_{1}$. Q.E.D.

We now show that the nonannihilating condition of Proposition 31 occurs with great frequency.

Proposition 32. Let $H$ be a hemiring. Then the condition $H_{1} H_{2} \neq 0$ defines 
an equivalence relation on the class of all nonzero minimal subtractive right ideals of $H$.

Proof. Clearly $H_{1}^{2} \neq 0$. If $H_{1} H_{2} \neq 0$, then $\left(H_{1} H_{2}\right)^{2}=H_{1}\left(H_{2} H_{1}\right) H_{2} \neq 0$, so that $H_{2} H_{1} \neq 0$. Now suppose $H_{1} H_{2} \neq 0$ and $H_{2} H_{3} \neq 0$. Then $H_{2} \&$ $\lambda\left(H_{3}\right)$, and by minimality, $H_{2} \cap \lambda\left(H_{3}\right)=0$. Since $H_{1} H_{2} \subseteq H_{2}, H_{1} H_{2} \cap \lambda\left(H_{3}\right)$ $=0$. Thus there are $h_{i} \in H_{i}$ with $h_{1} h_{2} h_{3} \neq 0$; hence $H_{1} \& \lambda\left(H_{3}\right)$. Q.E.D.

COROLlARY A. Let $H$ be the sum of nonzero minimal subtractive right ideals. If the sum of equivalent minimal subtractive right ideals is subtractive, it is a minimal subtractive two-sided ideal, and $H$ is the sum of these.

Proof. By using the (nonunique) representation of elements as a sum, it is quickly seen that the sum of equivalent minimal right ideals is a two-sided ideal, and that $H$ is the sum of these. Let $M=\Sigma H_{i}$ be such a two-sided ideal, and let $I \subset M$ be a subtractive two-sided ideal of $H$. Then $I \cap H_{1}=0$ for at least one of the $H_{i}$ 's $H_{1}$. Then for $\sum h_{i} \in I$, with $h_{i} \in H_{i}$, we have $\left(\sum h_{i}\right) H_{1}=\sum h_{i} H_{1}=0$. If $H_{1}$ is not a ring, $h_{i} H_{1}=0$ for each $i$, whence $I=0$. If $H_{1}$ is a ring, so also are all the $H_{i}$, and again $I=0$. Hence $M$ is minimal. Q.E.D.

Proposition 33. Let $H$ be a hemiring which is a strong direct sum of right ideals $H_{1}, \ldots, H_{n}$. Then if $(u, v)$ is any right unital pair for $H$, there exist right unital pairs $\left(u_{i}, v_{i}\right)$ in $H_{i}$ such that $u=\sum u_{i}, v=\sum v_{i}$. Each $H_{i}$ is subtractive, and $H_{i}=\left\{h \in H: h u_{i}+h=h v_{i}\right\}$. For $i \neq j, h u_{j}=h v_{j}$ for all $h \in H_{i}$.

Proof. It is clear that there exist $u_{i}, v_{i} \in H_{i}$ with $u=u=\Sigma u_{i}, v=\Sigma v_{i}$. For $h \in H, h u+h=h v$, and $\sum h v_{i}=h \sum v_{i}=h v=h+h u=h+h \sum u_{i}=$ $h+\sum h u_{i}$. If $h \in H_{j}, j \neq i$, it follows from the uniqueness of the representation that $h u_{i}=h v_{i}$, while $h u_{j}+h=h v_{j}$ and $\left(u_{j}, v_{j}\right)$ is a right unital pair for $H_{j}$. It is clear that each $H_{i}$ is subtractive, and that $H_{i}=\{h \in H$ : $\left.h u_{i}+h=h v_{i}\right\}$. Q.E.D.

We are now ready to obtain our matrix representation theorem.

THEOREM 34. Let $H$ be a simple halfring which is the strong direct sum of minimal subtractive right ideals $H_{1}, \ldots, H_{n}$ with primitive right unital pairs $\left(u_{i}, v_{i}\right)$. Let $P$ be the parafield $\left(v_{1}-u_{1}\right) H_{1}\left(v_{1}-u_{1}\right) \subseteq \bar{H}_{1}$. Then $H$ is isomorphic to a differential subring of $M_{n}(\bar{P})$.

Proof. Since $H$ is simple, $H_{i} H_{j} \neq 0$ for all $i, j$ by Proposition 32A, and there exist $S_{i j} \in e_{i} H e_{j}, S_{j i}^{\prime} \in e_{j} H e_{i}$ such that $S_{i j} S_{j i}^{\prime}=e_{i}, S_{j i}^{\prime} S_{i j}=e_{j}$, where $e_{i}=v_{i}-u_{i}$. Let $d_{i}=S_{i 1}, d_{i}^{\prime}=S_{1 i}^{\prime}$, so that $d_{i} d_{i}^{\prime}=e_{i}, d_{i}^{\prime} d_{i}=e_{1}$. For $h \in H$, define $h \phi \in M_{n}(\bar{P})$ by $[h \phi](i, j)=d_{i}^{\prime} h d_{j}=S_{1 i}^{\prime} h S_{j 1} \in e_{1} \bar{H} e_{i} h e_{j} H e_{1} \subseteq e_{1} \bar{P} e_{1}$. Clearly $\phi$ preserves addition. If $h, k \in H$, then 


$$
\begin{aligned}
{[(h k) \phi](i, j) } & =d_{i}^{\prime}(h k) d_{j}=d_{i}^{\prime} h e k d_{j} \\
& =d_{i}^{\prime} h\left(\sum_{m} e_{m}\right) k d_{j}=\sum_{m}\left(d_{i}^{\prime} h e_{m} k d_{j}\right)=\sum_{m}\left(d_{i}^{\prime} h d_{m} d_{m}^{\prime} k d_{j}\right) \\
& =\sum_{m}[h \phi](i, m)[k \phi](m, j)=[(h \phi)(k \phi)](i, j) .
\end{aligned}
$$

Hence $\phi$ is a homomorphism.

If $h \phi=k \phi$, then $d_{i}^{\prime} h d_{j}=d_{i}^{\prime} k d_{j}$ for all $i, j$. Hence $e_{i} h e_{j}=d_{i}\left(d_{i}^{\prime} h d_{j}\right) d_{j}^{\prime}=$ $d_{i}\left(d_{i}^{\prime} k d_{j}\right) d_{j}^{\prime}=e_{i} k e_{j}$, so that

$$
h=e h e=\left(\sum_{i} e_{i}\right) h\left(\sum_{j} e_{j}\right)=\sum_{i j} e_{i} h e_{j}=\sum_{i j} e_{i} k e_{j}=k .
$$

Hence $\phi$ is one-to-one.

Now $\phi: H \rightarrow M_{n}(\bar{P})$ extends to $\bar{\phi}: \bar{H} \rightarrow M_{n}(\bar{P})$, and $H \phi$ is differential in $M_{n}(P)$ if and only if $\bar{\phi}$ is onto. By Proposition 4, it suffices to show that $M_{n}(P) \subseteq \overline{H \phi}$. Let $p \in M_{n}(P)$, so that $p(i, j) \in P$ for all $i, j$. Let $x=$ $\Sigma_{i j} d_{i} p(i, j) d_{j}^{\prime} \in \bar{H}$. Then

$$
\begin{aligned}
{[x \bar{\phi}](r, s) } & =d_{r}^{\prime} x d_{s}=d_{r}^{\prime}\left(\sum_{i j} d_{i} p(i, j) d_{j}^{\prime}\right) d_{s} \\
& =\sum_{i j} d_{r}^{\prime} d_{i} p(i, j) d_{j}^{\prime} d_{s}=d_{r}^{\prime} d_{r} p(r, s) d_{s}^{\prime} d_{s} \\
& =e_{1} p(r, s) e_{1}=p(r, s) .
\end{aligned}
$$

Hence $x \bar{\phi}=p$, and $H \phi$ is differential in $M_{n}(\bar{P})$. Q.E.D.

We have paid a price for this generality. In the strongly Artinian case, we could say that our matrix representation was differential in the full matrix ring over a field; i.e., a right and left simple ring. Then the matrix ring itself was simple. Here we do not expect $M_{n}(\bar{P})$ to be simple. Hence instead of knowing that $H$ is differential in a simple matrix ring, we only know that $H$ is differential in a matrix ring which is the ring of differences of an even nicer matrix halfring.

Define halfrings $H_{1}$ and $H_{2}$ to be isodiffric if $\bar{H}_{1}$ is isomorphic to $\bar{H}_{2}$. Clearly this is an equivalence relation on the class of halfrings which is coarser than isomorphism. With this terminology, we may summarize our conclusion as follows: $H$ is isodiffric to the full halfring of matrices over a parafield.

\section{REFERENCES}

1. S. Bourne, On the multiplicative idempotents of a potent semiring, Proc. Nat. Acad. Sci. U.S.A. 42 (1956), 632-638.

2. S. Bourne and H. Zassenhaus, On a Wedderburn-Artin structure theory of a potent semiring, Proc. Nat. Acad. Sci. U.S.A. 43 (1957), 613-615. 
3. _ On the semiradical of a semiring, Pros. Nat. Acad. Sci. U.S.A. 44 (1958), 907-914.

4. M. P. Grillet and P. A. Grillet, Completely 0-simple semirings, Trans. Amer. Math. Soc. 155 (1971), 19-33.

5. M. P. Grillet, Semisimple A-semigroups and semirings, Fund. Math. 76 (1972), 109-116.

6. K. Iizuka, The Jacobson radical of a semiring, Tôhoku Math. J. 2 (1959), 409-421.

7. D. R. LaTorre, On h-ideals and $k$-ideals in hemirings, Publ. Math. Debrecen 12 (1965), 219-226.

8. O. Steinfeld, Über die Struktursatze der Semiringe, Acta Math. Acad. Sci. Hungar. 10 (1959), 149-155.

9. H. E. Stone, Ideals in halfrings, Proc. Amer. Math. Soc. 33 (1972), 8-14.

10. R. Wiegant, Über die Struktursatze der Halfringe, Ann. Univ. Sci. Budapest. Eötvös Sect. Math. 5 (1962), 51-68.

Department of Mathematics, University of Pittsburgh, Pittsburgh, Pennsylvania 15260

Current address: Department of Mathematics, University of Southwestern Louisiana, Lafayette, Louisiana 70504 